\title{
Direct Measurements of Deuterium Kinetic Isotope Effects in Anionic, Gas- Phase Substitution and Elimination Reactions
}

\author{
Scott Gronert, ${ }^{*}$ Adelaide E. Fagin, and Lawrence Wong \\ Department of Chemistry and Biochemistry, San Francisco State University, San \\ Francisco, CA 94132 USA.
}

\section{Supporting Information}

Table of Contents

1. Computational Details $\quad$ S1

2. Reference $13 \quad$ S1

3. Geometries and Energies of Alkyl Halides S2-S3

4. Geometries and Energies of Phenolate S4

5. Geometries and Energies of Phenolate TS's S4-S11

6. Geometries and Energies of Benzoate TS's S11-S13

\section{Computational Details}

All calculations were completed with Gaussian $03^{13}$ and include zero-point energies scaled by 0.9135 (Pople, J. A.; Scott, A. P.; Wong, M. W.; Radom, L., Isr. J. Chem. 1993, 33, 345). The frequencies were evaluated at the Hartee-Fock level. For the iodides, a 6-311+G(d) basis set was used in conjunction with Radom's all-electon basis set for iodine (Glukhovtsev, M.; Pross, A.; Mcgrath, M.; Radom, L., J. Chem. Phys. 1995, 103, 1878). For the bromides, a 6-31+G(d) basis set was used. Partition functions were evaluated at $298 \mathrm{~K}$ with no scaling factors. Reaction barriers were estimated for the bromides at the MP2/6-31+G(d,p)//MP2/6-31+G(d) level. The dianion nucleophiles are modeled by singly charged analogs, benzoate for I and phenolate for II. Barrier calculations were not pursued for the iodides due to known problems in modeling them computationally (Gronert, S.; Fagin, A. E.; Okamoto, K.; Mogali, S.; Pratt, L. M., J. Am. Chem. Soc. 2004, 126, 12977).

\section{Reference 13}

13. Frisch, M. J.; Trucks, G. W.; Schlegel, H. B.; Scuseria, G. E.; Robb, M. A.; Cheeseman, J. R.; Montgomery, J. A.; Vreven, T.; Kudin, K. N.; Burant, J. C.; Millam, J. M.; Iyengar, S. S.; Tomasi, J.; Barone, V.; Mennucci, B.; Cossi, M.; Scalmani, G.; Rega, N.; Petersson, G. A.; Nakatsuji, H.; Hada, M.; Ehara, M.; Toyota, K.; Fukuda, R.; Hasegawa, J.; Ishida, M.; Nakajima, T.; Honda, Y.; Kitao, O.; Nakai, H.; Klene, M.; Li, X.; Knox, J. E.; Hratchian, H. P.; Cross, J. B.; Adamo, C.; Jaramillo, J.; Gomperts, R.; Stratmann, R. E.; Yazyev, O.; Austin, A. J.; Cammi, R.; Pomelli, C.; Ochterski, J. W.; Ayala, P. Y.; Morokuma, K.; Voth, G. A.; Salvador, P.; Dannenberg, J. J.; Zakrzewski, V. G.; Dapprich, S.; Daniels, A. D.; Strain, M. C.; Farkas, O.; Malick, D. K.; Rabuck, A. D.; Raghavachari, K.; Foresman, J. B.; Ortiz, J. V.; Cui, Q.; Baboul, A. G.; Clifford, S.; Cioslowski, J.; Stefanov, B. B.; Liu, G.; Liashenko, A.; Piskorz, P.; Komaromi, I.; Martin, R. L.; Fox, D. J.; Keith, T.; Al-Laham, M. A.; Peng, C. Y.; Nanayakkara, A.; Challacombe, M.; Gill, P. M. W.; Johnson, B.; Chen, W.; Wong, M. W.; Gonzalez, C.; Pople, J. A. Gaussian 03, Revision B04; Gaussian, Inc.: Pittsburgh, PA, 2003. 


\section{All Energies in Hartee}

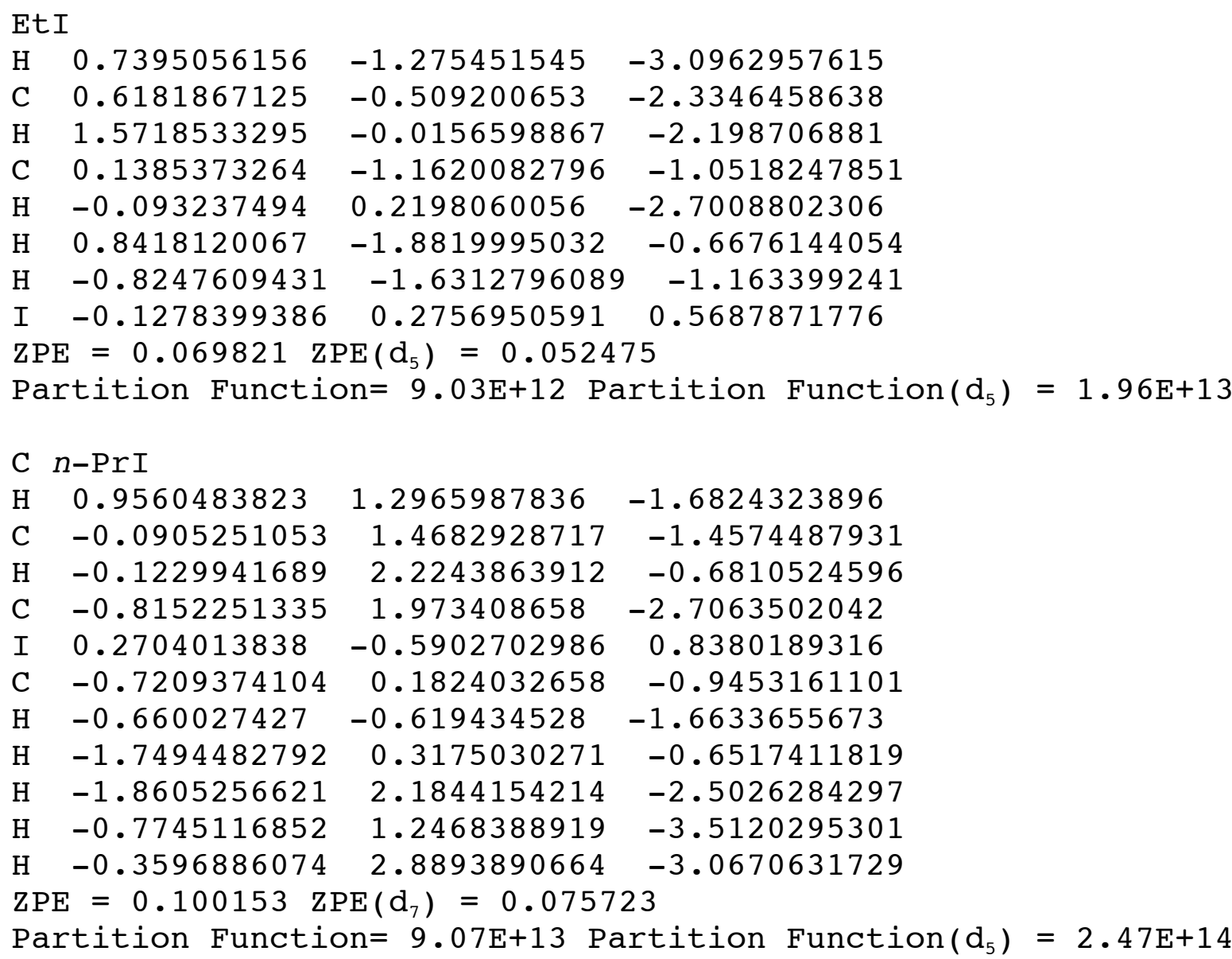

$\begin{array}{llcc}i-\operatorname{PrI} & & \\ \mathrm{H} & 1.9771642184 & 1.3019019094 & -1.0509861543 \\ \mathrm{C} & 1.8421815612 & 1.4224264186 & 0.0170934599 \\ \mathrm{H} & 2.8216026144 & 1.5745345404 & 0.4651263535 \\ \mathrm{C} & 1.203892778 & 0.1916844805 & 0.6399520188 \\ \mathrm{H} & 1.2522397473 & 2.3130285871 & 0.1877726754 \\ \mathrm{C} & 2.0158829867 & -1.0773326717 & 0.4390639375 \\ \mathrm{I} & -0.8327223076 & -0.086684805 & -0.170729562 \\ \mathrm{H} & 1.0100930538 & 0.3551499408 & 1.6881000075 \\ \mathrm{H} & 2.996463182 & -0.9414988007 & 0.8897832505 \\ \mathrm{H} & 2.1575822423 & -1.2954340427 & -0.612550511 \\ \mathrm{H} & 1.5473932905 & -1.9340568321 & 0.9047646678 \\ \mathrm{ZPE} & =0.099821 & \mathrm{ZPE}\left(\mathrm{d}_{7}\right)=0.075524\end{array}$

Partition Function $=5.92 \mathrm{E}+13$ Partition Function $\left(\mathrm{d}_{5}\right)=1.64 \mathrm{E}+14$

$\begin{array}{llll}n-\operatorname{PrBr} \mathrm{HF} & & \\ \mathrm{C} & 0.5373801298 & 0.3664643826 & 0.5147143647 \\ \mathrm{H} & 1.0623736197 & -0.2461711485 & 1.2306101487 \\ \mathrm{C} & -0.7066347455 & 1.0011239601 & 1.1096512783\end{array}$




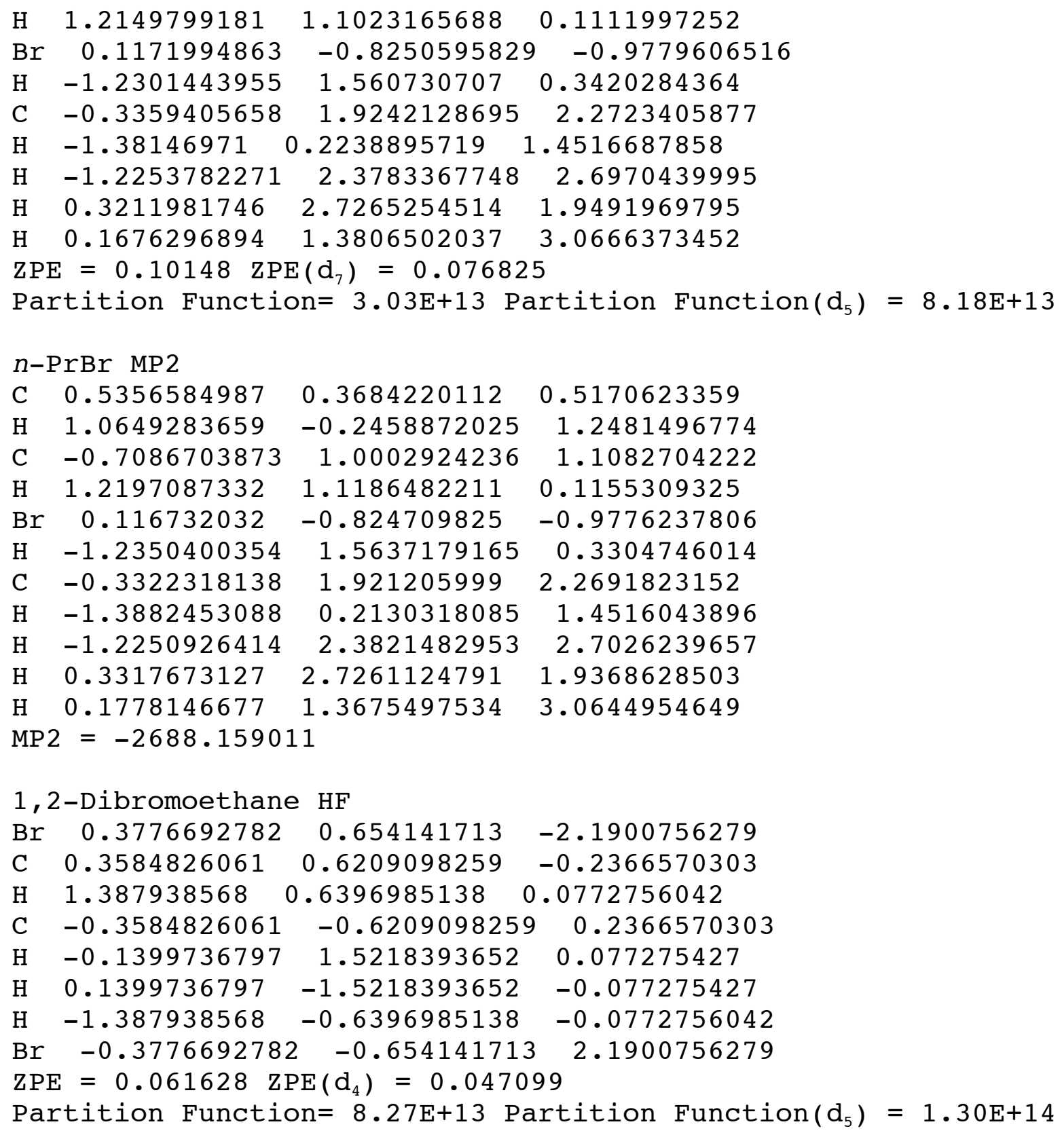

1,2-Dibromoethane MP2

$\begin{array}{llll}\text { Br } & 0.3722971469 & 0.6448369113 & -2.1929680534 \\ \mathrm{C} & 0.3585608517 & 0.6210453515 & -0.2349427968 \\ \mathrm{H} & 1.3995890756 & 0.6379840923 & 0.0873392706 \\ \mathrm{C} & -0.3585608517 & -0.6210453515 & 0.2349427968 \\ \mathrm{H} & -0.1472836651 & 1.5310717932 & 0.0873390912 \\ \mathrm{H} & 0.1472836651 & -1.5310717932 & -0.0873390912 \\ \mathrm{H} & -1.3995890756 & -0.6379840923 & -0.0873392706 \\ \mathrm{Br} & -0.3722971469 & -0.6448369113 & 2.1929680534 \\ \mathrm{MP} 2=-5218.400836 & & \end{array}$




\begin{tabular}{|c|c|c|c|}
\hline \multicolumn{4}{|c|}{ Phenolate MP2 } \\
\hline 0 & -1.255789968 & 1.8707370449 & 0.7110890829 \\
\hline $\mathrm{C}$ & -0.5713772814 & 0.851363625 & 0.3233902854 \\
\hline C & 0.9745763356 & -1.4516838329 & -0.5519586612 \\
\hline C & -1.1314514389 & -0.2167271131 & -0.4678008212 \\
\hline $\mathrm{C}$ & 0.8240791765 & 0.6740052669 & 0.6423405061 \\
\hline C & 1.560726629 & -0.4333658506 & 0.2187547926 \\
\hline $\mathrm{C}$ & -0.3840571745 & -1.3192031607 & -0.8852856711 \\
\hline $\mathrm{H}$ & -2.1857307301 & -0.1319389105 & -0.7369164143 \\
\hline & 1.2974140501 & 1.4546121768 & 1.2404408334 \\
\hline & 2.6159790572 & -0.5062697168 & 0.4952404257 \\
\hline & -0.8702380722 & -2.0942207712 & -1.4838613146 \\
\hline & 1.5539179611 & -2.3144127457 & -0.8802587764 \\
\hline
\end{tabular}

Phenolate with EtI E2

$\begin{array}{llll}\mathrm{H} & 0.6429751151 & -0.2505640576 & -0.6298389778 \\ \mathrm{C} & 0.0763071494 & 0.7209456453 & -0.0174002628 \\ \mathrm{H} & 0.8592161251 & 1.2107080173 & 0.5394913052 \\ \mathrm{C} & -0.7701163188 & -0.1237636632 & 0.6938383127 \\ \mathrm{H} & -0.3727869623 & 1.3276347084 & -0.787597012 \\ \mathrm{H} & -0.4136695322 & -0.647418413 & 1.5566817063 \\ \mathrm{H} & -1.6449733966 & -0.5305171134 & 0.2302181203 \\ \mathrm{I} & -2.5996624784 & 1.2121115487 & 2.5098991842 \\ \mathrm{O} & 1.1987454451 & -1.3640949814 & -1.2471571472 \\ \mathrm{C} & 2.1495922387 & -1.3743783758 & -2.1149285221 \\ \mathrm{C} & 4.2481328456 & -1.451253095 & -4.0343844108 \\ \mathrm{C} & 2.7606466316 & -0.1968805663 & -2.6000026692 \\ \mathrm{C} & 2.6397665911 & -2.5932812412 & -2.6373940337 \\ \mathrm{C} & 3.6575461739 & -2.621780184 & -3.5679620916 \\ \mathrm{C} & 3.7803745174 & -0.2448388416 & -3.5331089328 \\ \mathrm{H} & 2.4170487623 & 0.7538266425 & -2.2288118426 \\ \mathrm{H} & 2.191297998 & -3.5056930319 & -2.2845446876 \\ \mathrm{H} & 4.0000281161 & -3.5747186795 & -3.9393841161 \\ \mathrm{H} & 4.2176809417 & 0.6797309056 & -3.8751708144 \\ \mathrm{H} & 5.0418316547 & -1.4807592765 & -4.7603876031 \\ \mathrm{ZPE} & =0.161325 & \mathrm{zPE}\left(\mathrm{d}_{5}\right)=0.145799 \\ \mathrm{Partition} \text { Function }=9.19 \mathrm{E}+19 & \text { Partition } & \text { Function }\left(\mathrm{d}_{5}\right)=1.74 \mathrm{E}+20\end{array}$

Phenolate with EtI $\mathrm{S}_{\mathrm{N}} 2$

$\begin{array}{llll}\mathrm{H} & 1.5861109919 & 2.4892148575 & 0.8390523434 \\ \mathrm{C} & 1.0065035856 & 2.2428078927 & -0.0389014089 \\ \mathrm{H} & 0.0992273171 & 2.8324203797 & -0.0529982836 \\ \mathrm{H} & 1.5918973716 & 2.4560206357 & -0.92169214 \\ \mathrm{I} & 2.9373983899 & -0.4836356554 & 0.0189707854 \\ \mathrm{C} & 0.5651154862 & 0.8174242182 & -0.013472882 \\ \mathrm{H} & 0.3013208984 & 0.3119879209 & -0.9114278182 \\ \mathrm{H} & 0.2952736987 & 0.3462026729 & 0.9011381613\end{array}$




\begin{tabular}{|c|c|c|c|c|}
\hline $\mathrm{O}$ & -1.6151480633 & 1.4044843062 & -0.0317821277 & \\
\hline $\mathrm{C}$ & -2.62064936 & 0.6200217463 & 0.0204362455 & \\
\hline C & -4.8755783617 & -1.1339895966 & 0.0049241418 & \\
\hline $\mathrm{C}$ & -3.2139947482 & 0.131471269 & -1.2143432548 & \\
\hline C & -3.2226765144 & 0.1773512629 & 1.1869420567 & \\
\hline C & -4.3123580702 & -0.670171502 & 1.1891585603 & \\
\hline $\mathrm{C}$ & -4.3037477051 & -0.715671517 & -1.1920601705 & \\
\hline $\mathrm{H}$ & -2.7892188907 & 0.4487433759 & -2.1522410442 & \\
\hline $\mathrm{H}$ & -2.8046407744 & 0.5302763351 & 2.1150785647 & \\
\hline $\mathrm{H}$ & -4.7340258358 & -0.9778716251 & 2.1336192979 & \\
\hline H & -4.7186188174 & -1.0592835748 & -2.1271011288 & \\
\hline $\mathrm{H}$ & -5.7239419909 & -1.7963583329 & 0.0145126626 & \\
\hline & $=0.167113 \mathrm{Z}$ & $P E\left(d_{5}\right)=0.14967$ & & \\
\hline & ctition Functi & $\mathrm{on}=1.96 \mathrm{E}+19 \mathrm{~Pa}$ & rtition Function $\left(d_{5}\right)$ & $=3.99 \mathrm{E}+19$ \\
\hline & enolate with $n$ & -PrI E2 & & \\
\hline $\mathrm{H}$ & 0.7438134934 & 0.1500852804 & -1.340437117 & \\
\hline$C$ & -0.0232423777 & 0.2862961837 & -0.5918404218 & \\
\hline $\mathrm{H}$ & 0.6295072436 & 0.4365402358 & 0.4864498074 & \\
\hline C & -0.9034869767 & 1.5038740147 & -0.7783595252 & \\
\hline$I$ & -2.3806714053 & -2.299096233 & -1.8948870092 & \\
\hline C & -0.5437473647 & -0.8852191954 & -0.0661986974 & \\
\hline $\mathrm{H}$ & -1.3721574183 & -0.8572703936 & 0.6126634027 & \\
\hline $\mathrm{H}$ & 0.0376915604 & -1.7841131016 & -0.0630318011 & \\
\hline $\mathrm{H}$ & -1.5906896851 & 1.6209870605 & 0.0525024257 & \\
\hline $\mathrm{H}$ & -1.4883979645 & 1.4153235405 & -1.6883949016 & \\
\hline $\mathrm{H}$ & -0.3032338272 & 2.4047984527 & -0.8393472819 & \\
\hline $\mathrm{O}$ & 1.2828257957 & 0.6526366825 & 1.7315299545 & \\
\hline $\mathrm{C}$ & 2.1368565849 & 1.5691501274 & 2.0258121962 & \\
\hline $\mathrm{C}$ & 4.0159621119 & 3.5910750772 & 2.7176854904 & \\
\hline $\mathrm{C}$ & 2.2789348172 & 2.0348953399 & 3.3534770144 & \\
\hline $\mathrm{C}$ & 2.9833844055 & 2.1634731303 & 1.0633637179 & \\
\hline $\mathrm{C}$ & 3.891931683 & 3.1472624987 & .4085432706 & \\
\hline $\mathrm{C}$ & 3.1936256134 & 3.014411105 & .6808080915 & \\
\hline $\mathrm{H}$ & 1.6482086638 & 1.5975427535 & 4.1079531754 & \\
\hline $\mathrm{H}$ & 2.9142128065 & 1.8288563918 & 0.041805847 & \\
\hline $\mathrm{H}$ & 4.5176877141 & 3.5728820269 & 0.6401390708 & \\
\hline $\mathrm{H}$ & 3.2686085938 & 3.3384872377 & 4.7068328368 & \\
\hline $\mathrm{H}$ & 4.7264159534 & 4.3555777145 & 2.9798895672 & \\
\hline & $=0.191464 \mathrm{Z}$ & $P E\left(d_{7}\right)=0.16893$ & & \\
\hline & ctition Functi & $\mathrm{on}=1.16 \mathrm{E}+21 \mathrm{~Pa}$ & rrtition Function $\left(d_{5}\right)$ & $=2.98 \mathrm{E}+21$ \\
\hline & enolate with $n$ & $-\operatorname{PrI} \quad S_{N} 2$ & & \\
\hline $\mathrm{H}$ & 1.0213811162 & 0.6588929044 & -1.190130054 & \\
\hline $\mathrm{C}$ & 0.0957506668 & 1.1287419469 & -0.876831965 & \\
\hline $\mathrm{H}$ & 0.3683021465 & 2.007601602 & 0.3084586187 & \\
\hline $\mathrm{C}$ & -0.7438383027 & 1.4945686346 & -2.0936093885 & \\
\hline I & -2.1815471318 & 1.6462280465 & 1.5726949778 & \\
\hline
\end{tabular}




$\begin{array}{llll}\mathrm{C} & -0.5553684384 & 0.1342570666 & 0.0339434767 \\ \mathrm{H} & -0.0440741967 & -0.2004562709 & 0.9062421915 \\ \mathrm{H} & -1.396456566 & -0.4384779225 & -0.2728550527 \\ \mathrm{O} & 0.3145749263 & -1.7323460334 & -0.9481389609 \\ \mathrm{C} & 1.5456548762 & -2.0242483517 & -1.0742836934 \\ \mathrm{C} & 4.3204707545 & -2.6591041386 & -1.3540500628 \\ \mathrm{C} & 2.2619672569 & -1.788489572 & -2.2794109037 \\ \mathrm{C} & 2.3053040648 & -2.5970346212 & -0.0164548068 \\ \mathrm{C} & 3.6430684412 & -2.9001136562 & -0.1616851427 \\ \mathrm{C} & 3.601943186 & -2.1005072031 & -2.4047680238 \\ \mathrm{H} & 1.7205233559 & -1.3680110708 & -3.1106291598 \\ \mathrm{H} & 1.7979818412 & -2.8005679198 & 0.9119459872 \\ \mathrm{H} & 4.1738441464 & -3.3355698501 & 0.670892952 \\ \mathrm{H} & 4.0987275278 & -1.905325189 & -3.3427814667 \\ \mathrm{H} & 5.3645541395 & -2.8987084295 & -1.4588335933 \\ \mathrm{H} & -1.6691934076 & 1.9771767933 & -1.8012038628 \\ \mathrm{H} & -0.9824393225 & 0.6075674732 & -2.6699357665 \\ \mathrm{H} & -0.1974672361 & 2.1761390516 & -2.7390726302 \\ \mathrm{ZPE} & =0.197568 & \mathrm{ZPE}\left(\mathrm{d}_{7}\right)=0.173018\end{array}$

Partition Function $=7.07 \mathrm{E}+19$ Partition Function $\left(d_{5}\right)=1.73 \mathrm{E}+20$

Phenolate with i-PrI E2

$\begin{array}{llll}\text { H } & -0.0481600753 & 1.4797217032 & 2.08234942 \\ \mathrm{C} & -0.0915296523 & 0.4029707465 & 1.9993497381 \\ \mathrm{H} & 0.9112106688 & 0.0000167753 & 2.1311260796 \\ \mathrm{C} & -0.5234479044 & -0.0304786384 & 0.6473644027 \\ \mathrm{H} & -0.7417650497 & -0.00134416 & 2.7587629835 \\ \mathrm{C} & -0.1964256328 & 0.6555695694 & -0.5138864727 \\ \mathrm{I} & -3.7337568657 & 0.2094859524 & 1.154096224 \\ \mathrm{H} & -0.8779583154 & -1.0387681029 & 0.5523735209 \\ \mathrm{H} & 0.9473573869 & 0.1493626035 & -0.4975018119 \\ \mathrm{H} & -0.0385841069 & 1.719516252 & -0.43471271 \\ \mathrm{H} & -0.6673350537 & 0.3277333444 & -1.425007151 \\ \mathrm{O} & 2.2386844572 & -0.4736720245 & -0.2884039074 \\ \mathrm{C} & 3.2894532345 & -0.4296467617 & -1.0290197736 \\ \mathrm{C} & 5.6354145769 & -0.3594822693 & -2.638796471 \\ \mathrm{C} & 3.384727162 & 0.3903444526 & -2.1757259957 \\ \mathrm{C} & 4.4272767163 & -1.2133615281 & -0.7270880918 \\ \mathrm{C} & 5.5606589988 & -1.1731957278 & -1.5126548727 \\ \mathrm{C} & 4.5286657543 & 0.4171251621 & -2.952142506 \\ \mathrm{H} & 2.5404211317 & 1.0044861915 & -2.4410824301 \\ \mathrm{H} & 4.3826851464 & -1.848955928 & 0.1403948759 \\ \mathrm{H} & 6.4048706837 & -1.788587975 & -1.2444799139 \\ \mathrm{H} & 4.5557391475 & 1.0580281783 & -3.8190753938 \\ \mathrm{H} & 6.5223971412 & -0.3336581293 & -3.2474158235 \\ \mathrm{ZPE}=0.1912 \text { ZPE }\left(\mathrm{d}_{7}\right)=0.168755 & \\ \text { Partition Function }=1.44 \mathrm{E}+21 & \text { Partition Function }\left(\mathrm{d}_{5}\right)=3.83 \mathrm{E}+21\end{array}$




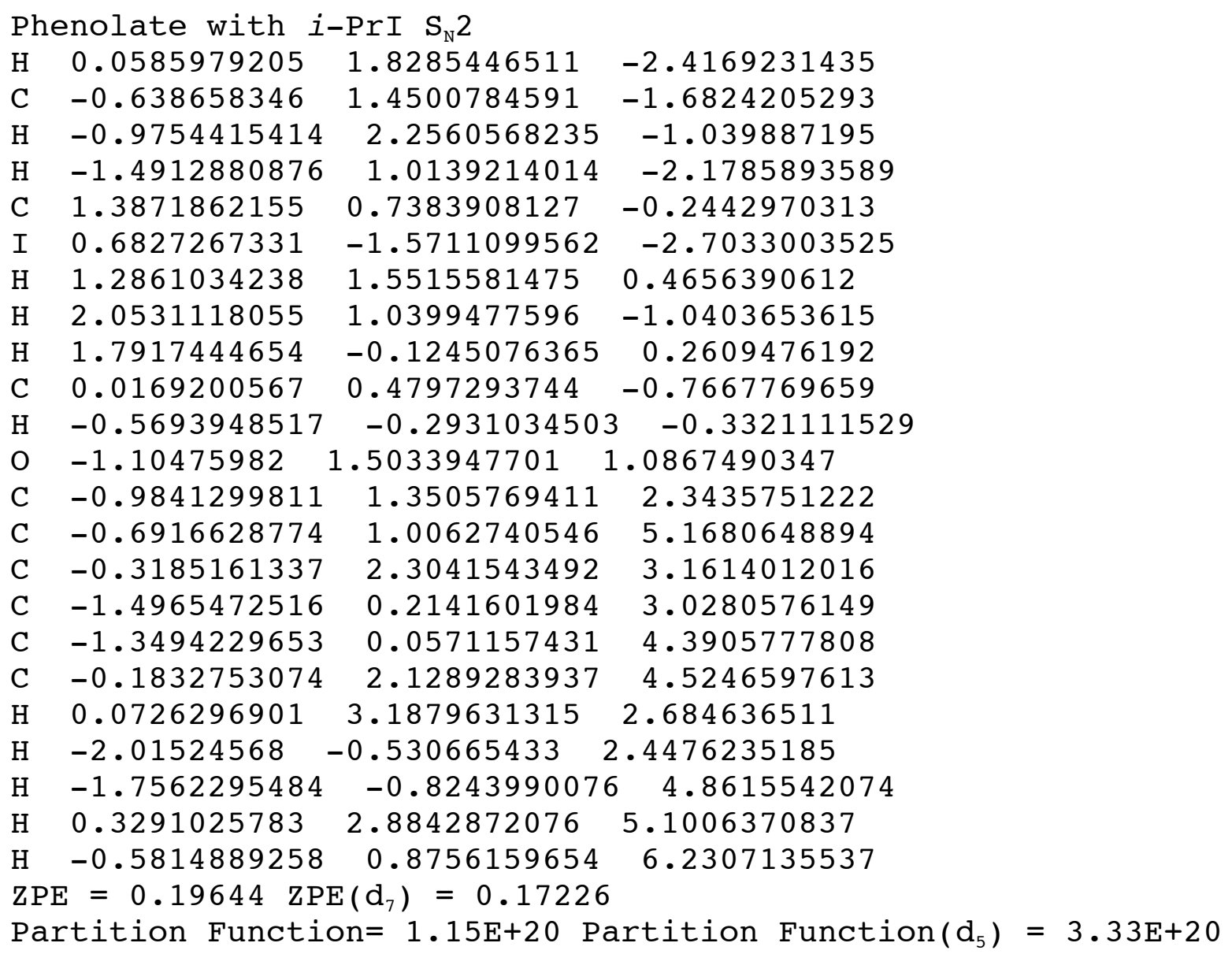

Phenolate with $n-\operatorname{PrBr} \mathrm{E} 2 \mathrm{HF}$

$\begin{array}{llll}\mathrm{C} & -1.688196616 & -0.4635122827 & -1.9127124479 \\ \mathrm{C} & -2.0890303922 & -1.0380982937 & -4.6692297664 \\ \mathrm{C} & -2.968553113 & -0.7594417109 & -2.433161551 \\ \mathrm{C} & -0.6178646084 & -0.4643565516 & -2.8339009341 \\ \mathrm{C} & -0.8238527661 & -0.7486608083 & -4.1736969197 \\ \mathrm{C} & -3.1555276841 & -1.037116552 & -3.7733781675 \\ \mathrm{H} & -3.7989095 & -0.7629756656 & -1.7486995154 \\ \mathrm{H} & 0.3726238873 & -0.2388432565 & -2.4794915057 \\ \mathrm{H} & 0.0213012852 & -0.7406366696 & -4.84337475 \\ \mathrm{H} & -4.149602678 & -1.2579588343 & -4.1285314138 \\ \mathrm{H} & -2.2401632275 & -1.2562612886 & -5.712245988 \\ \mathrm{O} & -1.5371037464 & -0.2067581009 & -0.6529256397 \\ \mathrm{H} & -0.3576014765 & -0.1168266836 & -0.0781774749 \\ \mathrm{C} & 0.8150067545 & -0.097778508 & 0.5618903312 \\ \mathrm{H} & 1.4576632753 & 0.4875118718 & -0.0833568726 \\ \mathrm{C} & 1.2481692686 & -1.5398163672 & 0.7453899376 \\ \mathrm{C} & 0.3016822115 & 0.603138449 & 1.6544931073 \\ \mathrm{Br} & 2.0278529922 & 1.2235784647 & 3.3992541576 \\ \mathrm{H} & 0.0803006367 & 1.6447399452 & 1.5549895691 \\ \mathrm{H} & -0.2604204107 & 0.0798154136 & 2.4013042427\end{array}$




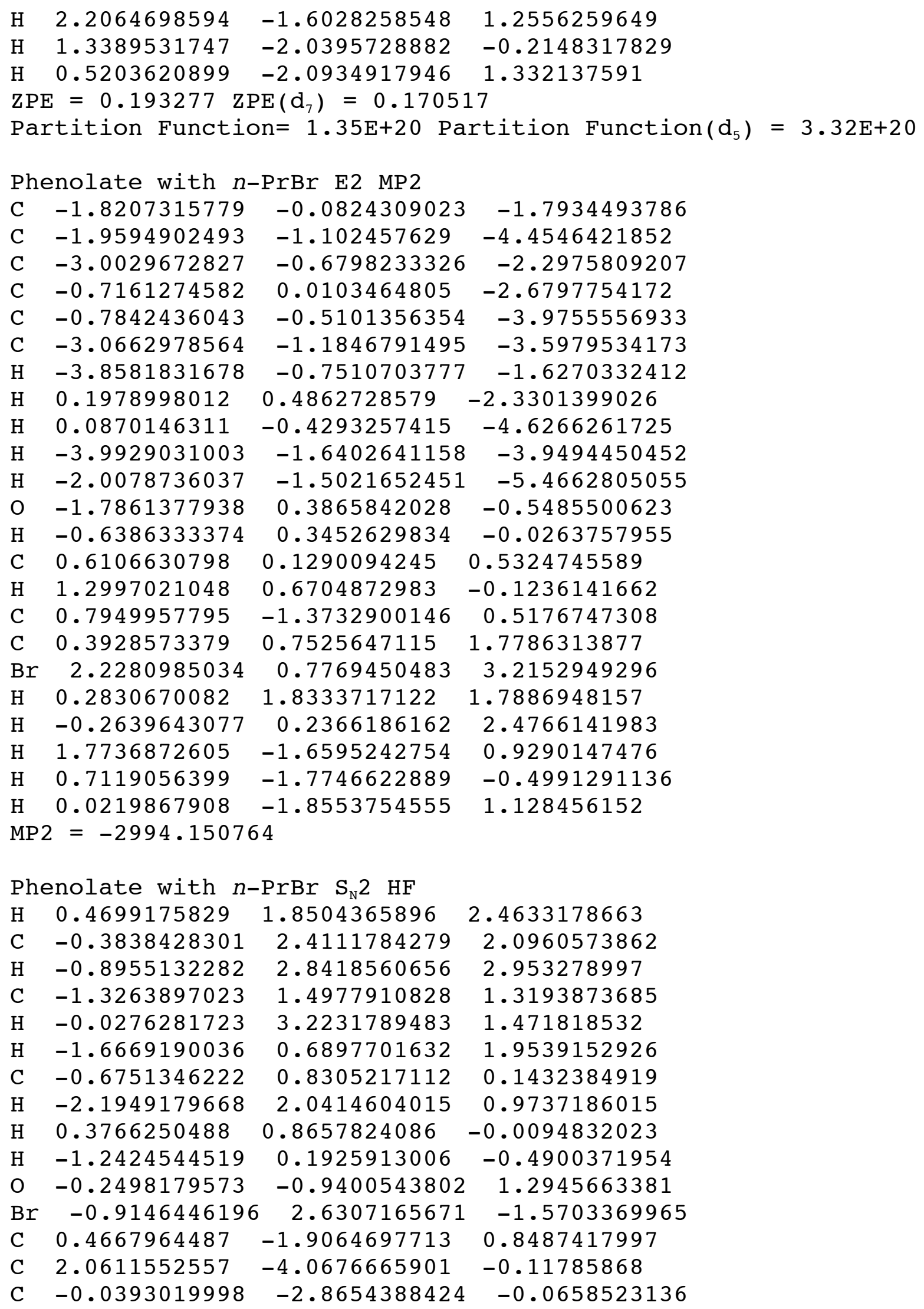




$\begin{array}{llll}\mathrm{C} & 1.8168720349 & -2.0842678127 & 1.2472158444 \\ \mathrm{C} & 2.5810460788 & -3.13587019 & 0.776905143 \\ \mathrm{C} & 0.7400175565 & -3.9106802148 & -0.527145667 \\ \mathrm{H} & -1.0625369114 & -2.7662127317 & -0.3876144302 \\ \mathrm{H} & 2.231579359 & -1.3751509188 & 1.943790934 \\ \mathrm{H} & 3.6023848916 & -3.2324220046 & 1.1110389912 \\ \mathrm{H} & 0.3110477863 & -4.6175748245 & -1.2200886845 \\ \mathrm{H} & 2.6622110895 & -4.8829470092 & -0.4825277699 \\ \mathrm{ZPE}=0.199726 & \mathrm{ZPE}\left(\mathrm{d}_{7}\right)=0.174892 \\ \text { Partition Function }=2.71 \mathrm{E}+19 & \text { Partition Function }\left(\mathrm{d}_{5}\right)=6.44 \mathrm{E}+19\end{array}$

Phenolate with $n-\operatorname{PrBr} \mathrm{S}_{\mathrm{N}} 2 \mathrm{MP} 2$

$\begin{array}{llcl}\mathrm{H} & 1.2783768519 & 1.5826239026 & 1.6495344853 \\ \mathrm{C} & 0.502618602 & 2.3392197134 & 1.4931913921 \\ \mathrm{H} & 0.5293348327 & 3.040467669 & 2.3360641873 \\ \mathrm{C} & -0.8591887352 & 1.6625973355 & 1.3854532304 \\ \mathrm{H} & 0.7259368759 & 2.8968695347 & 0.5772187609 \\ \mathrm{H} & -1.0610430576 & 1.0940535458 & 2.2994213916 \\ \mathrm{C} & -0.8966687686 & 0.6812030313 & 0.2441446978 \\ \mathrm{H} & -1.6538528713 & 2.4042325869 & 1.265612446 \\ \mathrm{H} & -0.0130905288 & 0.4749612067 & -0.3393171823 \\ \mathrm{H} & -1.7538956691 & 0.0440153598 & 0.1059284319 \\ \mathrm{O} & -0.1720594602 & -0.8019404746 & 1.4951979428 \\ \mathrm{Br} & -1.6790017297 & 2.0789672614 & -1.5156957682 \\ \mathrm{C} & 0.6920799723 & -1.6308699792 & 0.9478528353 \\ \mathrm{C} & 2.603668421 & -3.4475332759 & -0.1827571144 \\ \mathrm{C} & 0.5890013303 & -2.0916395751 & -0.3976517037 \\ \mathrm{C} & 1.8169263276 & -2.1016931791 & 1.6852689567 \\ \mathrm{C} & 2.7317976256 & -3.0053190555 & 1.1424453083 \\ \mathrm{C} & 1.5124068067 & -2.9939781453 & -0.934793384 \\ \mathrm{H} & -0.2650415379 & -1.7772762561 & -0.9959441252 \\ \mathrm{H} & 1.9195439521 & -1.7566776156 & 2.714054911 \\ \mathrm{H} & 3.5727363578 & -3.3445161458 & 1.7496935181 \\ \mathrm{H} & 1.3887057924 & -3.3274142061 & -1.9662514044 \\ \mathrm{H} & 3.3179757339 & -4.1515911542 & -0.6071723872 \\ \mathrm{H} 2 & =-2994.161817 & & \end{array}$

MP2 $=-2994.161817$

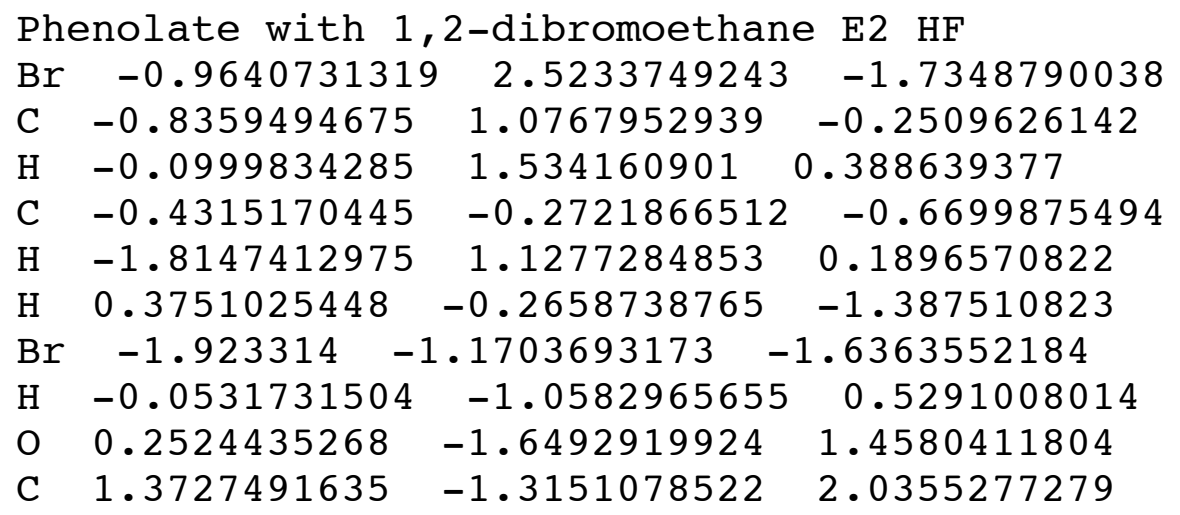




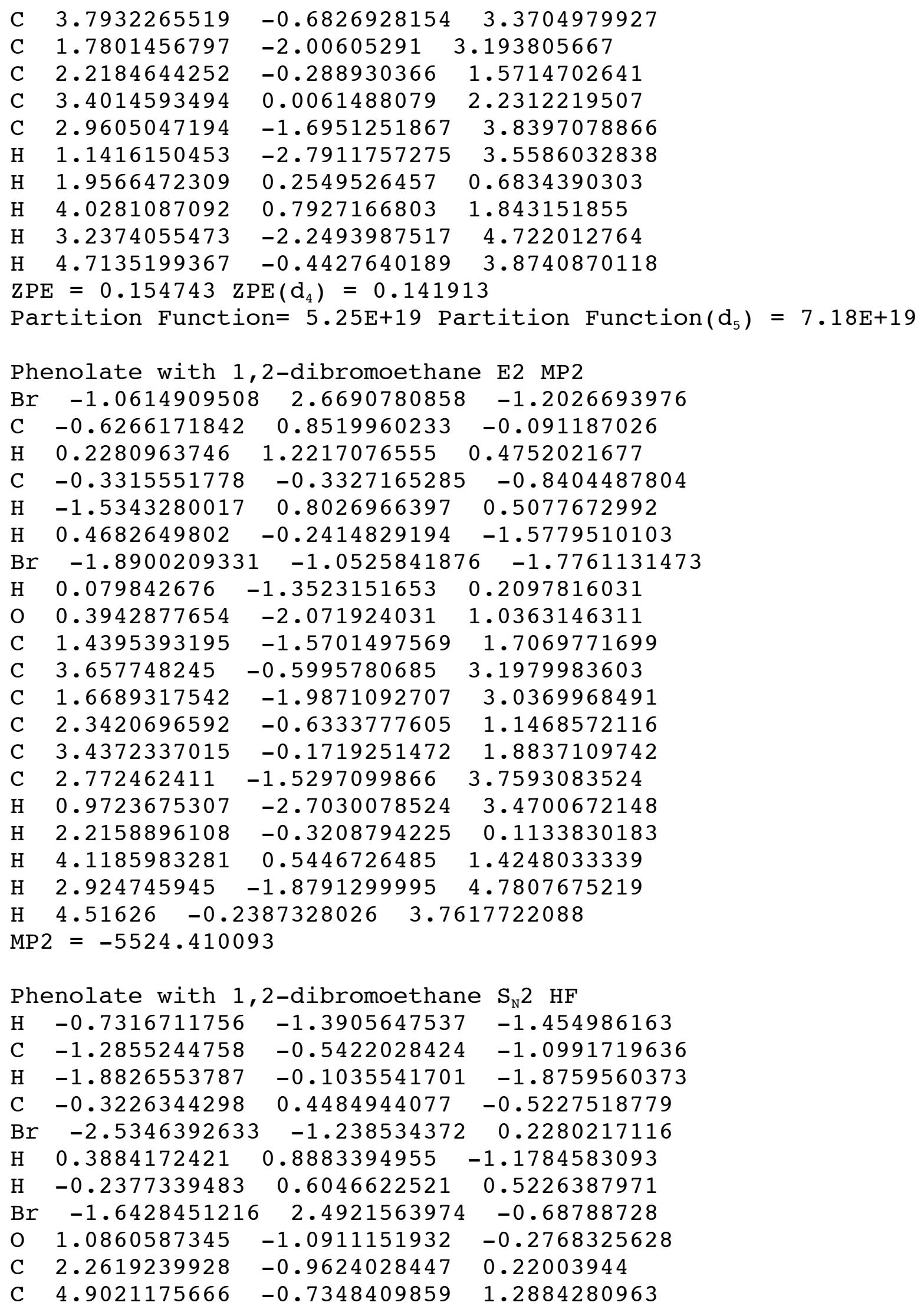




$\begin{array}{llll}\text { C } & 2.7849611879 & 0.2722443683 & 0.6791292978 \\ \mathrm{C} & 3.1347998564 & -2.0778898486 & 0.3242545162 \\ \mathrm{C} & 4.4086016753 & -1.9599023225 & 0.8407830654 \\ \mathrm{C} & 4.0661414724 & 0.3703357995 & 1.1967556672 \\ \mathrm{H} & 2.1715519694 & 1.1550047261 & 0.6251350908 \\ \mathrm{H} & 2.7669038907 & -3.0305660446 & -0.0164334944 \\ \mathrm{H} & 5.0329129757 & -2.837918365 & 0.8979412448 \\ \mathrm{H} & 4.4169167039 & 1.3329347813 & 1.5338158785 \\ \mathrm{H} & 5.8965202404 & -0.6492016532 & 1.691460941 \\ \text { ZPE = 0.161071 ZPE }\left(\mathrm{d}_{4}\right)=0.146304 \\ \text { Partition Function }=1.42 \mathrm{E}+19 & \text { Partition Function }\left(\mathrm{d}_{5}\right)=1.93 \mathrm{E}+19\end{array}$

Benzoate with i-PrI E2

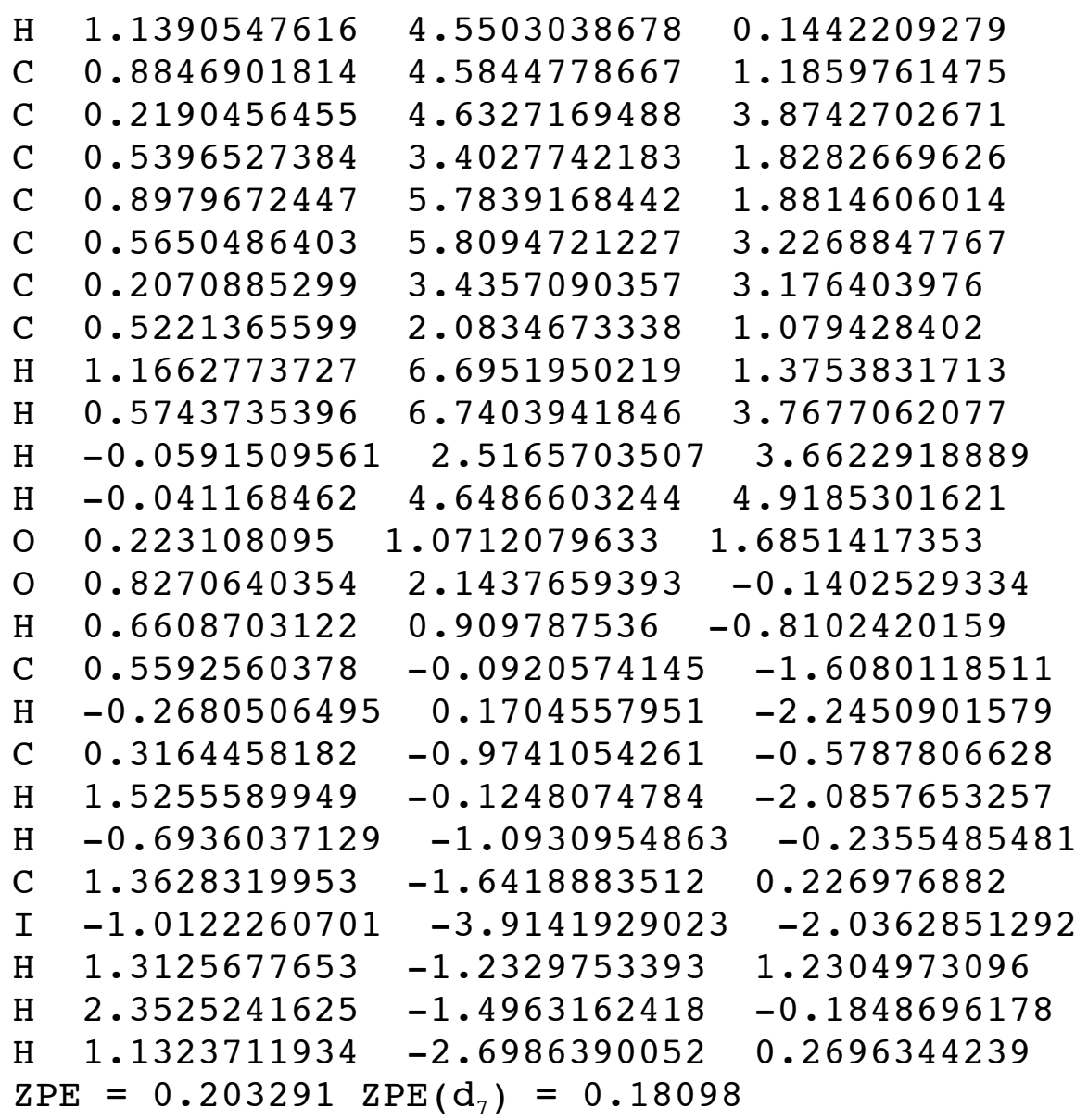

Partition Function= 5.57E+21 Partition Function $\left(d_{5}\right)=1.51 \mathrm{E}+22$ 


$\begin{array}{llll}\mathrm{C} & 4.2482076247 & -1.300368274 & -0.0106785345 \\ \mathrm{C} & 1.9273037925 & -0.3216950008 & 0.2348673493 \\ \mathrm{H} & 5.8040673018 & 2.1679090165 & -0.5843370384 \\ \mathrm{H} & 7.2386788716 & 0.1619256106 & -0.5942699195 \\ \mathrm{H} & 3.7952883037 & -2.2600925021 & 0.1495906799 \\ \mathrm{H} & 6.2347972381 & -2.0611043424 & -0.2269336862 \\ \mathrm{O} & 1.4960844836 & -1.450200063 & 0.4096218735 \\ \mathrm{O} & 1.272371692 & 0.7385303982 & 0.2299156637 \\ \mathrm{C} & -1.1296572072 & 0.1235291352 & 1.8233216594 \\ \mathrm{H} & -1.2193635751 & -0.9460142215 & 1.9165238435 \\ \mathrm{C} & -1.1118974156 & 0.5446408496 & 0.3961734776 \\ \mathrm{H} & -1.9275661926 & 0.6182778598 & 2.359384677 \\ \mathrm{H} & -0.8535366382 & -0.1724416969 & -0.346316744 \\ \mathrm{C} & -1.2462077023 & 1.9828732108 & 0.0313478136 \\ \mathrm{I} & -3.8561091198 & -0.2493142513 & -0.3171802196 \\ \mathrm{H} & -0.3072817451 & 2.4493483602 & 0.303338505 \\ \mathrm{H} & -2.0520092898 & 2.444305928 & 0.5838506241 \\ \mathrm{H} & -1.4125202188 & 2.1103610451 & -1.0267914881 \\ \mathrm{H} & -0.1757719501 & 0.4311575052 & 2.2356703291 \\ \mathrm{ZPE}=0.208816 \text { ZPE }\left(\mathrm{d}_{7}\right)=0.184626 & \\ \text { Partition Function }=2.31 \mathrm{E}+21 & \text { Partition Function }\left(\mathrm{d}_{5}\right)=6.74 \mathrm{E}+21\end{array}$

